\title{
Charge Transport Across Insulating Self- Assembled Monolayers: Non-equilibrium Approaches and Modeling To Relate Current and Molecular Structure
}

\section{Citation}

Mirjani, Fatemeh, Joseph M. Thijssen, George M. Whitesides, and Mark A. Ratner. 2014. “Charge Transport Across Insulating Self-Assembled Monolayers: Non-Equilibrium Approaches and Modeling To Relate Current and Molecular Structure." ACS Nano 8 (12) (December 23): 1242812436. doi:10.1021/nn505115a.

\section{Published Version}

doi:10.1021/nn505115a

\section{Permanent link}

http://nrs.harvard.edu/urn-3:HUL.InstRepos:16922666

\section{Terms of Use}

This article was downloaded from Harvard University's DASH repository, and is made available under the terms and conditions applicable to Open Access Policy Articles, as set forth at http:// nrs.harvard.edu/urn-3:HUL.InstRepos:dash.current.terms-of-use\#OAP

\section{Share Your Story}

The Harvard community has made this article openly available.

Please share how this access benefits you. Submit a story. 


\title{
Charge transport across insulating self-assembled
}

\author{
monolayers: Non-equilibrium approaches and \\ modeling to relate current and molecular
}

\section{structure}

Fatemeh Mirjani, ${ }^{* \dagger}$ Joseph M. Thijssen, ${ }^{\ddagger}$ George M. Whitesides, $₫$ and Mark A. Ratner $^{\S}$

E-mail: fateme.mirjani@gmail.com

*To whom correspondence should be addressed

${ }^{\dagger}$ Chemical Engineering Department, Delft University of Technology, Julianalaan 136, 2628 BL Delft, The Netherlands

${ }_{\ddagger}^{\ddagger}$ Kavli Institute of Nanoscience, Delft University of Technology, Lorentzweg 1, 2628 CJ Delft, The Netherlands

`Department of Chemistry and Chemical Biology, Harvard University, 12 Oxford, Cambridge, Massachusetts, 02138, USA; and Kavli Institute for Bionano Science and Technology, Harvard University, 29 Oxford, Cambridge, Massachusetts, 02138, USA

$\S$ Department of Chemistry and Non-Equilibrium Research Center, Northwestern University, 2145 Sheridan Road, Evanston, Illinois, 60208-3113, USA 


\begin{abstract}
This paper examines charge transport by tunneling across a series of electrically (insulating molecules with the structure $\mathrm{HS}\left(\mathrm{CH}_{2}\right)_{4} \mathrm{CONH}\left(\mathrm{CH}_{2}\right)_{2} \mathrm{R}$ ) in the form of selfassembled monolayers (SAMs), supported on silver. The molecules examined were studied experimentally by Yoon et al. (Angew. Chem. Int. Ed., 51, 4658-4661, 2012), using junctions of the structure $\mathrm{AgS}\left(\mathrm{CH}_{2}\right)_{4} \mathrm{CONH}\left(\mathrm{CH}_{2}\right)_{2} \mathrm{R} / / \mathrm{Ga}_{2} \mathrm{O}_{3} / \mathrm{EGaIn}$. The tail group $\mathrm{R}$ had approximately the same length for all molecules, but a range of different structures. Changing the $\mathrm{R}$ entity over a range of different structures (aliphatic to aromatic) does not influence the conductance significantly. To rationalize this surprising result, we investigate transport through these SAMs theoretically, using both full quantum methods and a generic, independent-electron tight-binding toy model. We find that the frontier orbitals HOMO, HOMO-1, HOMO-2 and HOMO-3, have similar structures for all of the different R-groups, and that the HOMO, which is largely responsible for the transport in these molecules, is always strongly localized on the thiol group. The relative insensitivity of the current density to the structure of the $\mathrm{R}$ group is due not only to these similar frontier orbitals but also to a combination of energy levels $(\varepsilon)$ and tunneling amplitudes $(t)$, which lead to similar conductance for different tail groups, i.e. the large difference between $\varepsilon$ 's in conjugated and saturated groups is compensated by tunneling amplitudes inside the tail group. In addition, the coupling of the tail group to the alkane chain is much weaker for conjugated groups than for saturated ones. Conductance change in these molecules is not influenced by the broadening of the molecular levels connected to the left and right electrodes, since in all these molecules the effective coupling to the silver substrate is the same, whereas the other effective couplings are determined by the weak connection to the oxide $\mathrm{Ga}_{2} \mathrm{O}_{3}$ layer. All these factors combine to produce currents largely insensitive to the $\mathrm{R}$ group. An estimation of the attenuation constant, $\beta$, of the simplified Simmons equation obtained using these methods is $\beta=1.1$ per $\mathrm{CH}_{2}$ group, in good agreement with observed results. Our analysis sketches the potential landscapes across which tunneling occurs, and suggests that it will be difficult to change rates of tunneling by simple changes
\end{abstract}


in molecular structure. This work indicates that significant control over SAMs largely composed of nominally insulating groups may be possible when tail groups are used that are significantly larger than those used in the experiments of Yoon et al. ${ }^{13}$

Keywords: molecular electronics, self-assembled monolayers, molecular structure, charge transport, hole tunneling, current density 


\section{Introduction}

The putative field of 'Molecular Electronics' involves charge transport by processes in which the structure of the molecule forming the junction influences the conductance. Ultimately it hopes to design molecules whose electrical conductance can be tuned rationally through organic synthesis. ${ }^{1-7}$ One of the motivations for this field has been the expectation that small changes in the structure or the environment of the molecules would change the characteristics of charge transport through them in ways that might be useful in practical electronics, sensing, or controls. In single molecule devices with weak electrode/molecule interaction, Coulomb blockade enables switching the current on and off by a gate field. ${ }^{8,9}$ This type of control is, however, not the only way to control the current. Fracasso et al. ${ }^{10}$ showed that the conductance in anthracene derivatives of approximately the same thickness can be influenced by the type of conjugation. The results in that experiment ${ }^{10}$ were attributed to quantum interference, but the origin of the effect remains to be validated in other experiments. There are now many reports of substantially different conductance values measured in break junctions, see e.g. ref. ${ }^{11}$ Another example is the exponential decay of the conductance in alkane self-assembled monolayers (SAMs), which does not exist in n-polyene chains where a chain of $\mathrm{C}-\mathrm{C}$ single bonds $\left(\mathrm{CH}_{2}\right)_{2 n}$ is replaced by an extended conjugated chain $\left(\mathrm{CH}=\mathrm{CH}_{2}-\right)_{n} \cdot{ }^{12}$

Yoon et al., ${ }^{13}$ reported a systematic experimental study in SAMs which suggested that large changes in molecular structure (e.g. changing a cyclohexyl group for a phenyl group) need not induce significant changes in the conductance of molecular monolayers. Yoon's study also indicated that replacing ${ }^{13}-\mathrm{CH}_{2} \mathrm{CH}_{2}-$ in the interior of the molecules making up the SAMs by - CONH - had no effect on tunneling current. More specifically, they measured the current densities through a series of SAMs based on different molecules located between a silver electrode and $\mathrm{Ga}_{2} \mathrm{O}_{3} /$ EGaIn electrode, where EGaIn denotes eutectic gallium and indium (a liquid metal alloy ${ }^{14}$ ) and $\mathrm{Ga}_{2} \mathrm{O}_{3}$ is a spontaneously formed, electrically conducting surface oxide layer (normally $0.7 \mathrm{~nm}$ thick) on the EGaIn electrode. The structure of the molecules making up the SAMs is $\mathrm{HS}\left(\mathrm{CH}_{2}\right)_{4} \mathrm{CONH}\left(\mathrm{CH}_{2}\right)_{2} \mathrm{R}$ where $\mathrm{R}$ is one of the tail groups 


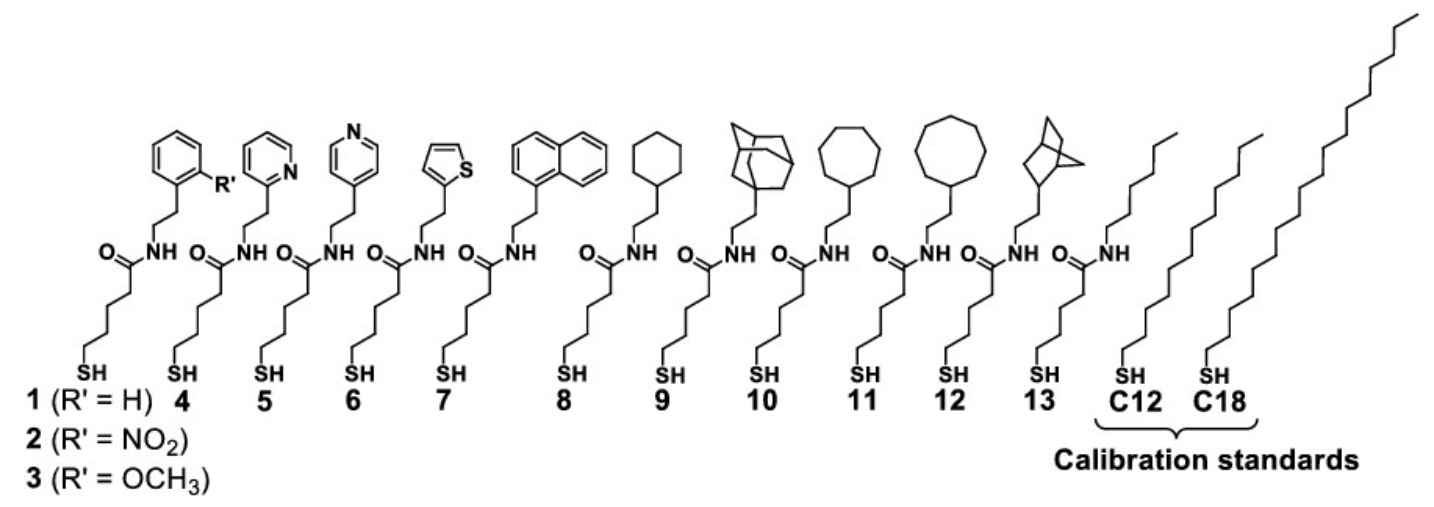

Figure 1: Structure of the molecules used by Yoon et al. ${ }^{13}$ to form the self-assembled monolayers on the Ag surface. The structure of all molecules 1-13 is $\mathrm{HS}\left(\mathrm{CH}_{2}\right)_{4} \mathrm{CONH}\left(\mathrm{CH}_{2}\right)_{2}-\mathrm{R}$ where $\mathrm{R}$ is the tail group. In the measurements, molecules $\mathrm{C} 12$ and $\mathrm{C} 18$ were presented as calibration standards. All current densities were measured through Ag-molecule- $\mathrm{Ga}_{2} \mathrm{O}_{3} /$ EGaIn structures.

shown in Fig. 1. In all these molecules, the tail groups have almost the same length. The tail structures can be divided into two groups: (1) aromatic structures, and (2) saturated aliphatic groups.

It is not obvious why SAMs of these very different molecules do not yield very different conductivities. ${ }^{1}$ The measured current densities for all molecules at $V_{\text {bias }}=0.5 \mathrm{~V}$ are shown in Fig. 2 on a logarithmic scale, with simple alkane chains of length 12 and 18 alkane units (e.g. $\left.\mathrm{S}\left(\mathrm{CH}_{2}\right)_{n-1} \mathrm{CH}_{3}\right)$ included. Alkane chains are standard molecules in which electron transport has been studied extensively. ${ }^{15-22}$ The main result of those prior studies is that the current decays exponentially with chain length with a decay constant $\beta \sim 1.0$ per $\mathrm{CH}_{2}$ unit, with some variation across different experiments. ${ }^{15-19,23}$ This dependence has been also successfully addressed computationally using Density Functional Theory (DFT) combined with non-equilibrium Green's function (NEGF) studies. ${ }^{16,22,24}$ Yoon et al. measured $\beta \sim 0.9$ per $\mathrm{CH}_{2}$ unit - a value that is in agreement with these earlier results. In fact, the 12- and 18-unit alkane chains are used as calibration standards in Yoon's experiment, to compare with the results obtained with the other molecules.

\footnotetext{
${ }^{1}$ Examination of the values of the current density in Fig. 2 suggests that aromatic tail groups might be slightly (2 times) more conducting than aliphatic ones; we have however taken the conservative point of view - based on the statistically well-defined standard deviation - that statistically we cannot distinguish aliphatic and aromatic tail groups.
} 


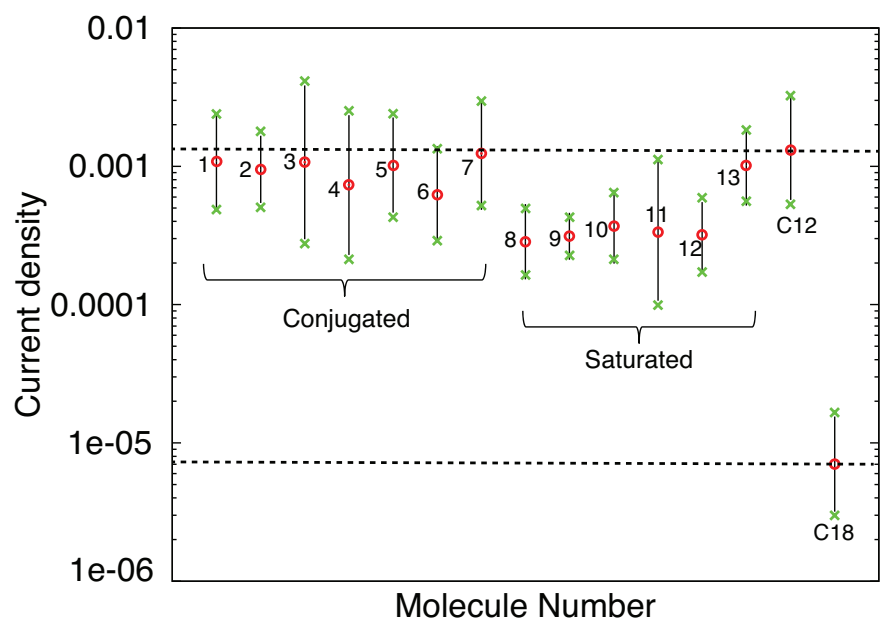

Figure 2: Current density measured by Yoon et al. ${ }^{13}$ at $V_{\text {bias }}=0.5 \mathrm{~V}$ for the molecules of Fig. 1 (presented on a logarithmic scale). The unit of the current density is $A / \mathrm{cm}^{2}$. The green crosses $(x)$ show the interval of the errors. Although the saturated structures (molecules 8-13) seem to have a somewhat smaller conductance than the conjugated ones (molecules 1-7), the differences are not significant within the experimental error.

Understanding the relatively constant current density observed for this complicated system is difficult, as different mechanisms may be responsible for the observed current densities. While inter-chain tunneling of the electrons may be significant, here we focus on the effects of the molecular electronic structure on the single-molecule current. The standard way to address this subject is by performing DFT-NEGF calculations. ${ }^{25,26}$ We did such calculations, which confirm that varying the tail group does not induce dramatic differences in the molecular conductance. To understand this result, we describe the transport using a simple tight-binding model, with parameters inferred from a series of ground-state DFT calculations. We call this the tight-binding toy model (TBTM). The procedure we follow is in the spirit of semi-empirical models which aim to explain experiments using parameters obtained via fitting to ab initio calculations or to experimental data. In particular, one of the criteria we have used to adjust the parameters is that the structure of a few of the highest occupied frontier orbitals, as obtained by full DFT calculations, is essentially reproduced in TBTM. The transmission of the model is then analyzed again using Green's function methods. This provides insight into why the current varies only weakly across the set. These results may 


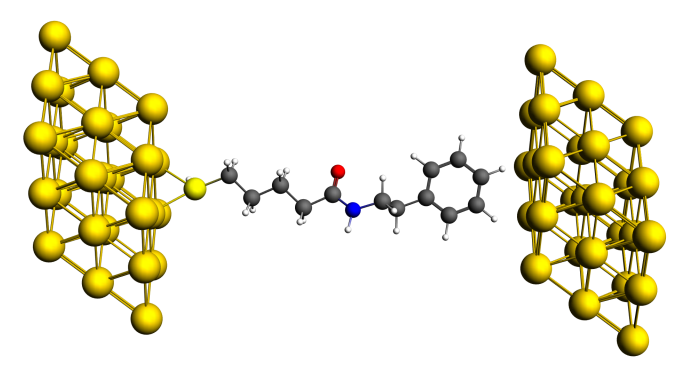

Figure 3: The structure of the extended molecule 1 in which the molecule is connected to $3 \times 3 \times 3$ Au clusters from left and right.

help designing future experiments to synthesize molecules with substantially different transmissions.

\section{DFT calculations}

\section{(A) DFT-NEGF based transport calculations}

We start with the transmission for molecules 1-13 obtained from DFT-NEGF. We have calculated the transmission through these molecules with two different methods:

(I) Gas phase-NEGF: In this method, DFT calculations for molecules in gas phase (thiolended, without electrodes) are performed and then the contribution of the molecule to transport is calculated by computing $\left|G_{1 N}{ }^{2}\right|$ between the $\mathrm{p}_{z}$ - orbitals (perpendicular to the plane through $\mathrm{S}, \mathrm{C}$ and $\mathrm{H}$ ) of the $S(1)$ and the $\mathrm{R}$ groups $(N)$ where $G$ denotes the Green's function and $\mathrm{S}$ is the sulfur atom of the thiol linker. ${ }^{27} G_{1 N}$ can be understood as the quantity which measures the tunneling amplitude from site 1 to site N. Once the Green's functions are known, the transmission can be obtained using the Landauer-type equation. Here we include self-energies within wide-band limit approximation. ${ }^{28}$

The coupling of the tail groups to the $\mathrm{Ga}_{2} \mathrm{O}_{3}$ oxide layer (on EGaIn electrode) is an unknown parameter which is not included in the gas-phase calculations. This method does not yield the correct location of the transmission peaks (of the occupied orbitals) since the molecules are considered in gas phase - shifts induced by interface dipoles and image charges 
are therefore not taken into account. To obtain better insight into these shifts, we have used another method.

(II) Extended molecule-NEGF: In this method, an extended molecule is used. The extended molecule is made by connecting the molecule to $3 \times 3 \times 3$ metal clusters from right and left. Then we connect this extended molecule to two electrodes which are $3 \times 3 \times 9$ clusters. For silver, we need to incorporate 19 electrons per silver atom for the largest frozen core, as opposed to 11 electrons per gold atom. In view of the similarity between the two contact types, ${ }^{29}$ and since that we are looking for trends which are expected to be the same for silver and gold, we have chosen to perform our calculations using gold contacts. Experimentally, with a metal alloy and a conductive oxide layer on one side of the molecule, the screening cannot be expected to let surface effects decay within the width of the contacts. We present the results for a molecule between two gold contacts as another reference calculation, with the awareness that the actual system (Ag on one side and oxide/metal on the other side) is somewhere in between that of the gas phase and the extended molecule with gold contacts.

To calculate the self-energies for each $3 \times 3 \times 9$ cluster, we divide the cluster into three layers where each layer consists of three sub-layers, as shown in Fig. 3. Then we calculate the transport properties of the system. This method relies on the strong screening in the contact regions, which renders the results relatively insensitive to their size.

The code used here is an in-house developed add-on to the Amsterdam Density Functional (ADF) $\operatorname{code}^{30}$ (using an LDA exchange correlation functional and a double-zeta polarized basis set). ${ }^{27,30}$ The Fermi energy of the gold electrode in our calculations is $-5.3 \mathrm{eV}$, which is taken as the midpoint between the HOMO and the LUMO of the $3 \times 3 \times 9$ Au cluster. The transmission results for both methods are shown in Fig. 4. In all molecules 1-13, the transmission peaks below the Fermi energy are closer to the Fermi energy than the peaks above the Fermi energy, indicating that the transport is hole-like. The influence of the metal in the calculation is reflected in the shift and broadening of transmission peaks. By integrating over the transmission curves of the first method we obtain the currents shown in 
(a)

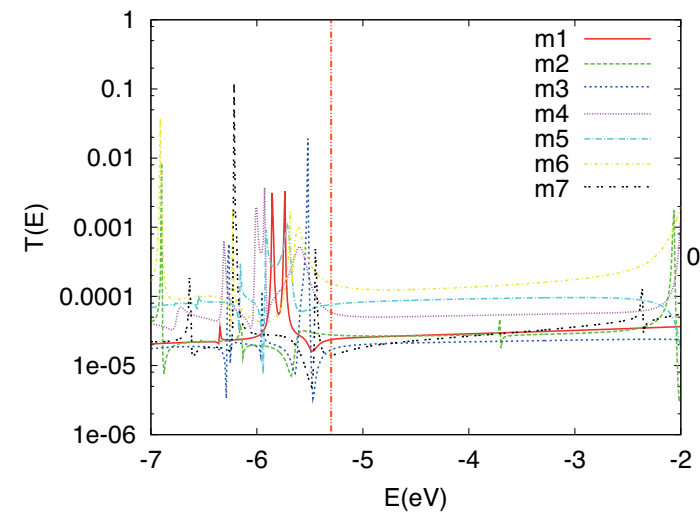

(c)

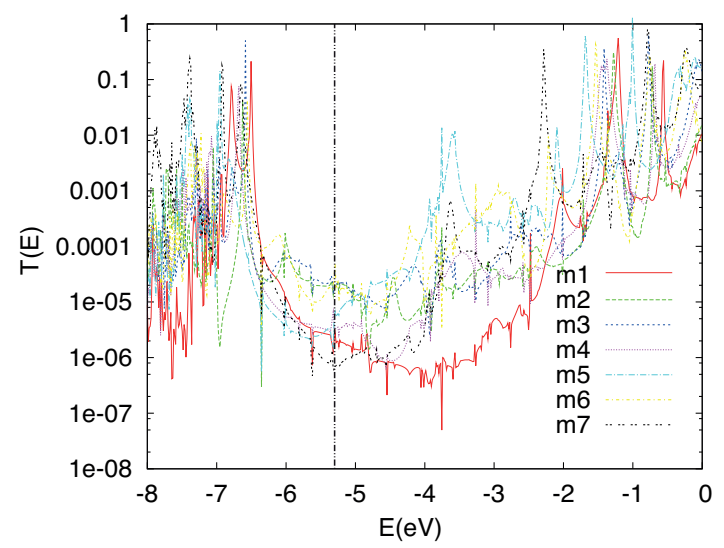

(b)

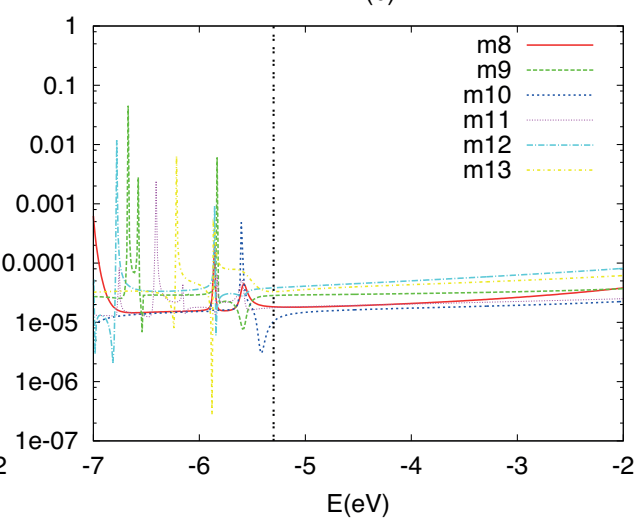

(d)

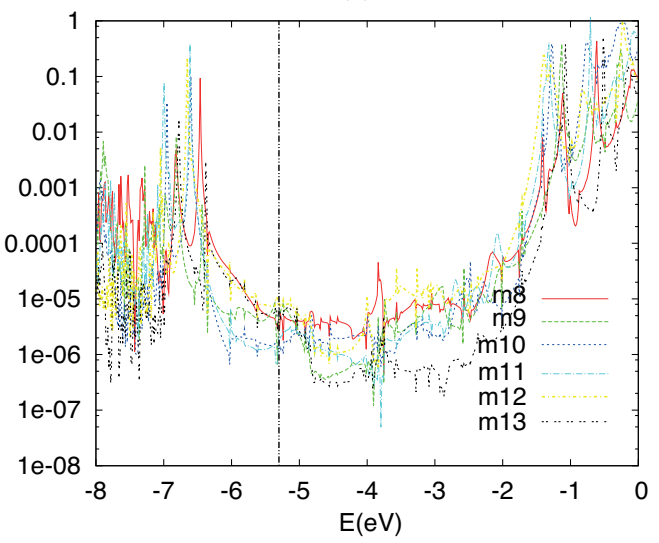

Figure 4: Transmission through molecules 1-7 (with conjugated tail groups) and molecules 8-13 (with saturated tail groups) on a logarithmic scale. The vertical dashed lines show the Fermi energy at $-5.3 \mathrm{eV}$. (a,b) Gas phase-NEGF, (c,d) Extended molecule-NEGF where the extended molecules consist of molecules and two $3 \times 3 \times 3$ Au clusters.

Fig. 5 which do not differ by more than a factor of 8 . In the next sections, we will develop a toy model which explains why the current through these structures shows only a modest variation.

\section{(B) Molecular orbitals}

We have performed ground state DFT calculations for the molecules in gas phase. The results give insight into the behavior of the electrons participating in transport, and help us to construct the TBTM Hamiltonian describing the landscape in which the electrons are 


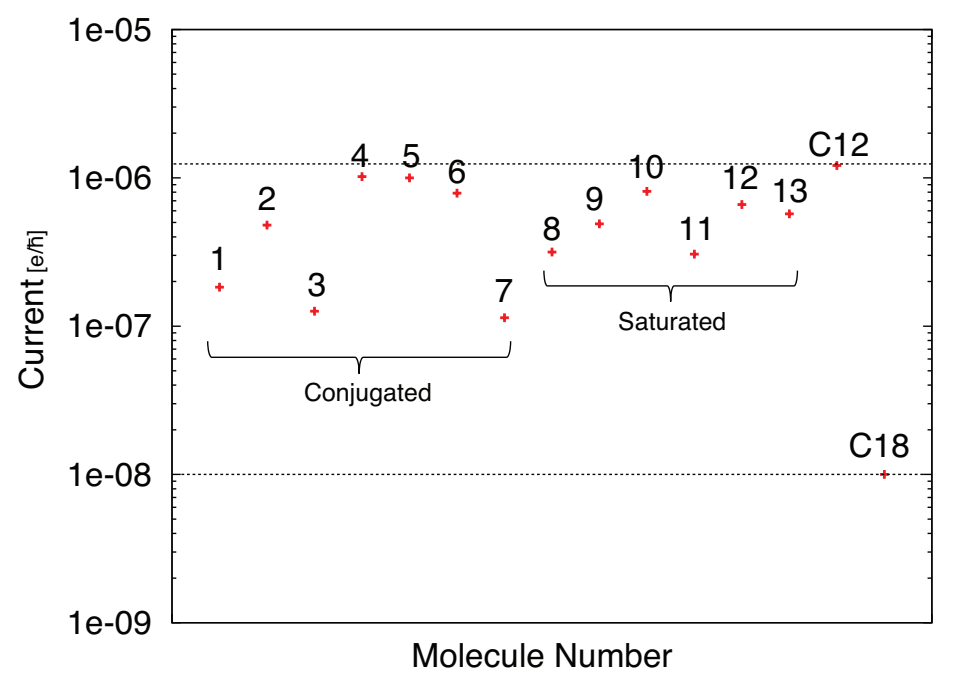

Figure 5: Current (shown on a logarithmic scale) obtained from gas phase-NEGF method. These results clearly show that the variations in transmission through these different molecules are modest, in agreement with the results for the current obtained by Yoon et $a l .{ }^{13}$

moving. DFT calculations for molecules 1-13 using the quantum chemistry codes ADF 30 and Q-chem ${ }^{31}$ were performed with a TZP (triple Zeta polarization) basis set, and a GGA exchange-correlation functional $(\mathrm{PBE})$ in $\mathrm{ADF}$, and $6-31 \mathrm{G}^{* *}$ basis set and PBE0 exchange correlation functional in Q-chem. Both codes give similar results - here we show those obtained using ADF.

From these results, we observe the following:

(I) The HOMO orbital for all molecules is localized on the sulfur atom bonded to the Ag surface in the experiment. The HOMO and LUMO orbitals are shown in Fig. 6 (HOMO stands for the highest occupied molecular orbital and LUMO denotes the lowest unoccupied molecular orbital). Both the HOMO energy and its shape are approximately the same for all molecules.

(II) The LUMO structure and energy vary quite strongly with tail group. The shape of the LUMO furthermore depends quite sensitively on the geometrical optimization. With our optimized geometries (that may or may not agree in detail with the structures found 
in the SAMs, which are not known in detail experimentally, and probably depend on the topography of the electrode surface), the LUMO of the molecules with saturated tail groups is located on the thiol group just as the HOMO, whereas for molecules with a conjugated tail group, it is located on the tail group.

(III) HOMO-1, HOMO-2, HOMO-3 of molecules 1-13 are mostly located on the amide, on both the amide and the tail group $\mathrm{R}$, and on the tail group $\mathrm{R}$ respectively; see supplementary information.

As the structure and chemical potential of the HOMO does not change substantially across different molecules, and as the HOMO is substantially closer to the Fermi energy of the electrodes $\left(E_{F}(A g)=-4.7 \mathrm{eV}, E_{F}\left(\mathrm{Ga}_{2} \mathrm{O}_{3} / E G a I n\right)=-4.3 \mathrm{eV}^{13}\right)$ than the LUMO, we expect the transport to be hole-like - that is, dominated through the HOMO, in agreement with our NEGF calculations.

\section{Tight-binding Model}

The DFT-based NEGF calculations just presented do reproduce the experimentally observed insensitivity of the current to the chemical composition of the tail group. To obtain a better understanding of this result, and to analyze the junction with a non-metallic electrode, we construct a simple tight-binding toy model (TBTM). The model Hamiltonian is designed such that the electrons and their dynamics reflect the behavior of electrons described by a full DFT Kohn-Sham Hamiltonian, as used in section II. This implies that the TBTM parameters, especially those which influence the energetics of low-lying occupied states, do not necessarily reflect spectral features (which are known to be badly reproduced by DFT). The guiding principle in constructing the TBTM model is that it produces reliable HOMO's, as the transport in the molecules under study is hole-like and off-resonant (see section II).

The probability for electron tunneling is low enough to neglect two electrons tunneling at the same time, and we can safely neglect spin. This junction is then represented by a 
( I ) With conjugated tails

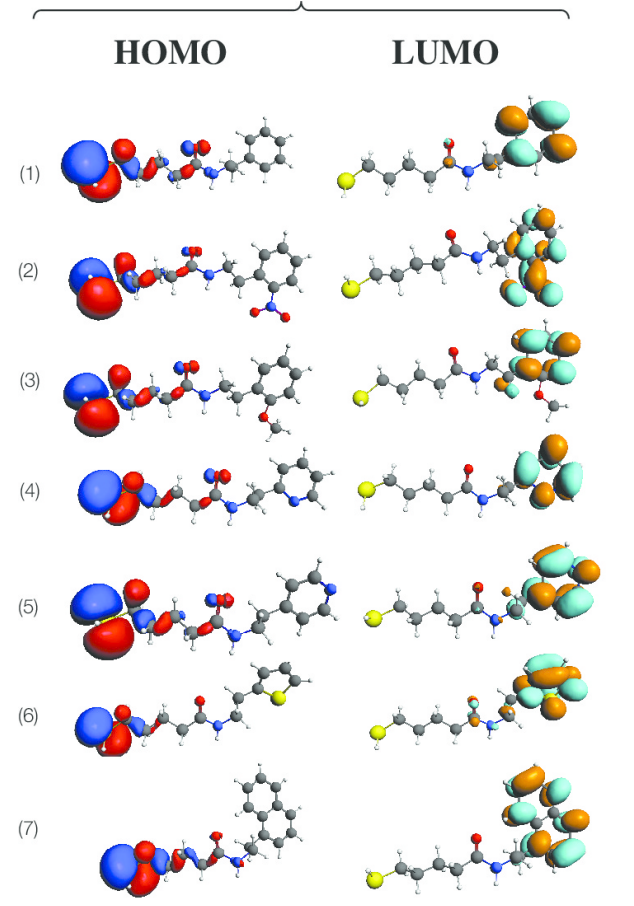

( II ) With saturated tails

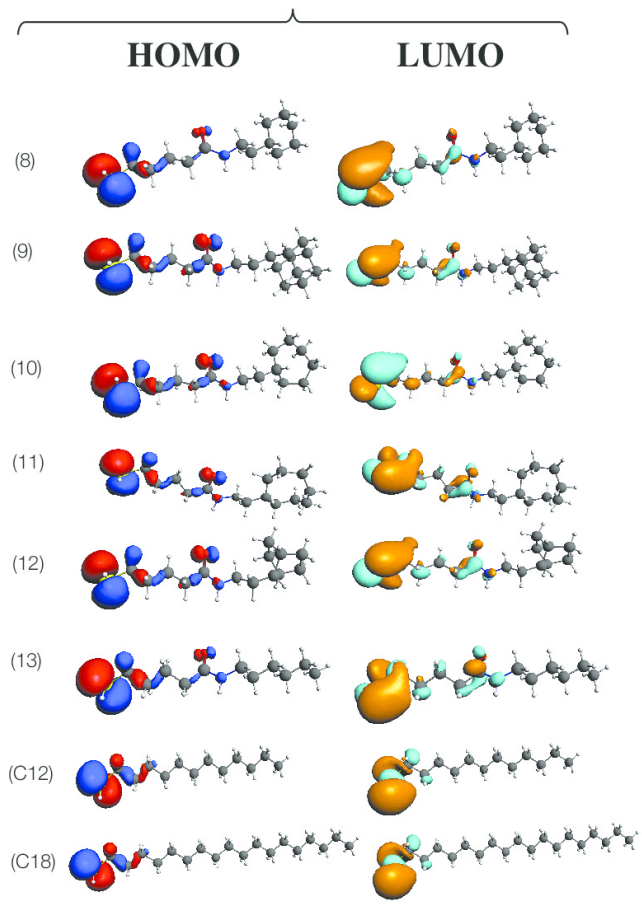

Figure 6: HOMO and LUMO of the molecules 1-13 from the DFT calculation. Molecules C12 and C18 were presented in the experiment as calibration standards. The HOMO of all of these molecules is largely localized on the thiol linker. The LUMO is localized on the tail group in molecules $1-7$, which have conjugated tails. However, in molecules $8-13$ with saturated tails, the LUMO is mostly localized on the thiol group.

simple, essentially linear tight-binding system, coupled to two non-interacting semi-infinite leads. The (extended Hückel-like) Hamiltonian ${ }^{32}$ of the molecular region reads

$$
H_{\text {molecule }}=-\sum_{\substack{i, j \\ i<j}} t_{i, j}\left[d_{i}^{\dagger} d_{j}+\text { H.c. }\right]+\sum_{i=1}^{N} \varepsilon_{i} d_{i}^{\dagger} d_{i}
$$

where H.c. denotes the Hermitian conjugate and $N$ is the length of the interacting chain. The parameters $t_{i, j}$ represent the tunneling amplitudes between the atomic orbitals $\phi_{i}$ and $\phi_{j}$ within the molecule. The electron creation and annihilation operators, $d_{i}^{\dagger}$ and $d_{j}$ satisfy the usual anti-commutation relations. The second term in the Hamiltonian represents the site energies $\varepsilon_{i}$.

The Schrödinger equation then takes the form $\mathrm{HC}_{\alpha}=E_{\alpha} \mathrm{SC}_{\alpha}$ where $\mathrm{C}_{\alpha}$ is a vector with 


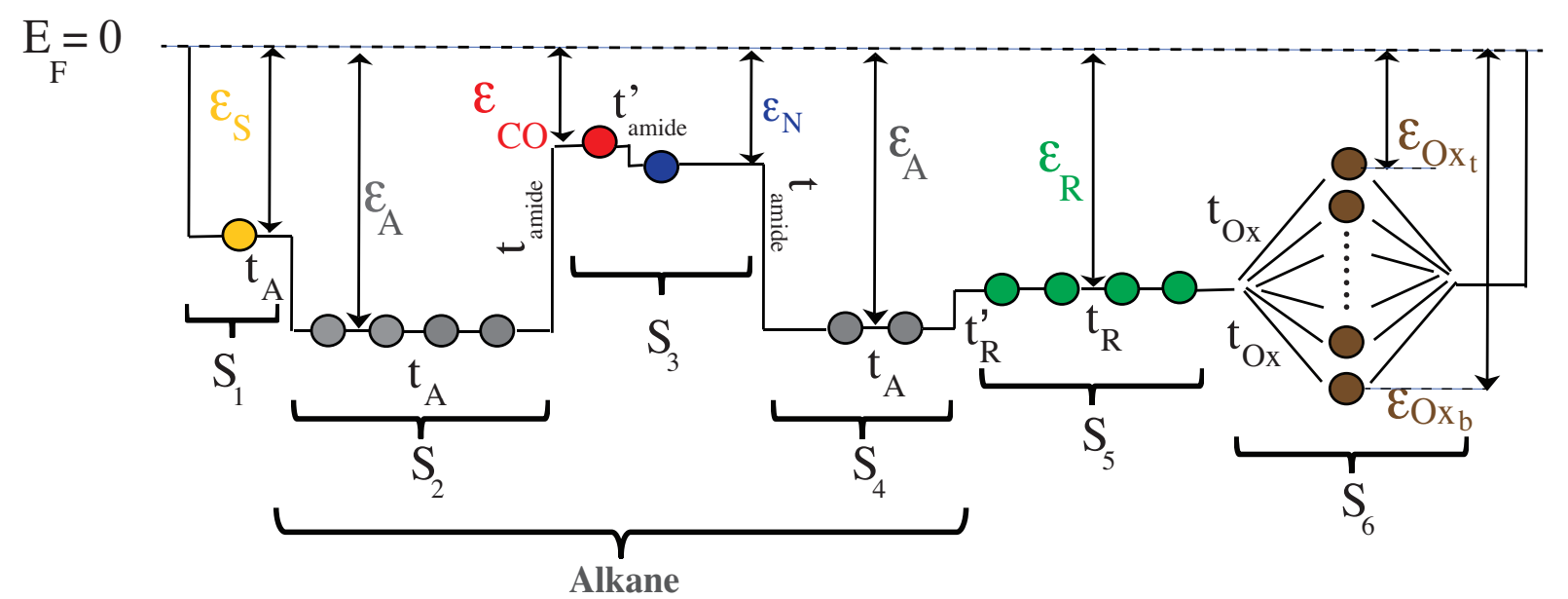

Figure 7: A tight-binding toy model (TBTM) structure representing the molecules 1-13 and the oxide layer. We model the molecular junction with six segments $S_{1}-S_{6}$. The barriers with different site energies represent the following parts of the molecules : $\varepsilon_{S}$ : thiol group. $\varepsilon_{\mathrm{A}}$ : alkane chain in which the energy of the central sites $\left(\varepsilon_{\mathrm{CO}}\right.$ and $\left.\varepsilon_{\mathrm{N}}\right)$ are chosen to be higher than the rest of subunits; they represent the amide group (CONH). $\varepsilon_{R}$ : tail group $R$ (which varies in molecules $1-15)$. $\varepsilon_{\mathrm{Ox}}: \mathrm{Ga}_{2} \mathrm{O}_{3}$ layer.

coefficients corresponding to $\phi_{i}$ such that the molecular orbital $\psi_{\alpha}=\sum_{i} C_{i, \alpha} \phi_{i}$, and $S$ is the overlap matrix. When the orbitals are normalized such that the diagonal elements of the overlap matrix are 1, then the off-diagonal elements between the neighboring sites are typically $\sim 0.3 .{ }^{33}$ Our analysis shows that the overlap matrix does not play an important role, and the results that we present below turn out to be insensitive to such deviation from orthonormality. Therefore, we will take $S$ to be the unit matrix (e.g, $S_{i i}=1, S_{i j}=0$ for $i \neq j$ ) in the remainder of the analysis. ${ }^{34}$

We want the tight-binding chain in our toy model to mimic the electronic properties of molecules 1-15 (Fig. 7). These molecules consist of six segments. The first segment (S1) is the thiol linker and the next three segments are $\mathrm{S} 2\left(\mathrm{CH}_{2}\right)_{4}$, S3 $\mathrm{CONH}$ and $\mathrm{S} 4\left(\mathrm{CH}_{2}\right)_{2}$ respectively. The next part (S5) is the tail group $\mathrm{R}$ and the last segment (S6) is the oxide layer. We find most of the parameters of the TBTM Hamiltonian of equation 1 from the results shown in Fig. 6 and Fig. 12, and from calculations on periodic chains, as we outline in the supporting information.

Our generic tight-binding chain for molecules 1-13 is shown in Fig. 7. It consists of 17 
sites, divided into six subunits. The first molecular subunit $\left(S_{1}\right)$, represents the thiol binding group connected to the silver electrode. Its site energy, $\varepsilon_{\mathrm{S}}$, is higher than that of the alkane chain. The following three groups of sites $\left(S_{2}, S_{3}, S_{4}\right)$, represent the alkane chain with the amide. The amide sites $\left(S_{3}\right)$ have energies $\varepsilon_{\mathrm{CO}}$ and $\varepsilon_{\mathrm{N}}$ that are higher than alkanes. It should be noted that our model presents a coarse-grained description of the molecules under study. In particular, in our description of the amide units, the $(\mathrm{C}=\mathrm{O})$ group is considered as one site, and the $\left(\mathrm{NH}_{2}\right)$ as another one.

Two amide subunits $(\mathrm{C}=\mathrm{O}$ and $\mathrm{NH})$ are coupled to neighbouring $\mathrm{CH}_{2}$ by $t_{\text {amide }}-$ we take those to be the same. The coupling inside the amide group is $t_{\text {amide }}^{\prime}$. The fourth substructure $S_{4}$, represents the two methylene sites between amide and $\mathrm{R}$. The tail group $\mathrm{R}$ (which varies in molecules $1-13)$ is denoted as $S_{5}$ and couples to the alkane part with $t_{\mathrm{R}}^{\prime}$. As the size of $\mathrm{R}$ is approximately the same in molecules $1-13$, we fix the length of $S_{5}$ to four sites. The last subunit, $S_{6}$, represents the $\mathrm{Ga}_{2} \mathrm{O}_{3}$ layer. This subunit includes a series of uncoupled sites which mimic the band structure of the oxide layer. The top site with energy level $\varepsilon_{\mathrm{Ox}_{\mathrm{t}}}$ represents the top of the band of this layer and similarly the parameter $\varepsilon_{\mathrm{Ox}_{\mathrm{b}}}$ corresponds to the bottom of the band. All the sites in this subunit are coupled to the last site of the tail group by $t_{\mathrm{Ox}}$. The only parameters that change across molecules 1-13 are the site energy and the tunneling parameters describing $S_{5}$, i.e. $\varepsilon_{\mathrm{R}}, t_{\mathrm{R}}$ and $t_{\mathrm{R}}^{\prime}$, where $t_{\mathrm{R}}^{\prime}$ describes the coupling to the $\mathrm{CH}_{2}$ group.

The orbitals found in DFT are evaluated with a potential corresponding to the neutral molecule. Our method is in the spirit of semi-empirical models like extended Hückel, ${ }^{35}$ PPP $,{ }^{36} \mathrm{CNDO},{ }^{37} \mathrm{MNDO}^{38}$ etc., which fit the tight-binding parameters to ab initio calculations or to experimental data.

The tight-binding parameters $\varepsilon$ and $t$ for each subunit are shown in Table. 1. In Hückel molecular orbital theory, these parameters are called $\alpha$ and $\beta$ respectively. Reproducing the structures of the orbitals found (see Fig. 6 and Fig. 12) is the most important criterion for finding the parameters. We match the parameters of the TB chain to those orbital 
Table 1: Tight-binding parameters $\varepsilon$ and $t$ for each subunit (in eV). Subunits of the same kind are coupled by $t$. The coupling of a subunit to a saturated $\mathrm{C}$ neighbour is $t_{\mathrm{n}}$, provided the subunit itself is not a saturated $\mathrm{C}$ (including methylene). Literature parameters are presented from (a) Benkö et al. ${ }^{39}$ (b) Xu et al. ${ }^{40}$ (c) Wang et al. ${ }^{41}$ (d) Murrell ${ }^{42}$ and (e) Salem. ${ }^{43} t$ parameter for the last subunit is zero as it includes a series of uncoupled sites which mimic the band structure of the oxide layer. All sites in this subunit are coupled to the last site of the tail group by $t=0.1$. For details, see supplementary information.

\begin{tabular}{|c|c|c|c|c|c|c|}
\hline Subunit & Site Number & Represent & $\varepsilon$ & $t, t_{\mathrm{n}}$ & $\varepsilon$ (literature) & $t$ (literature) \\
\hline$S_{1}$ & 1 & thiol & -4.2 & 6,6 & - & - \\
$S_{2}$ & $2-5$ & methylenes & -14 & 6 & $-13.9^{a}$ & $6^{b}, 7.7^{c}$ \\
$S_{3}$ & $6-7$ & amide & $-1.3,-1.8$ & $0.5,1.8$ & - & $1.8^{d}$ \\
$S_{4}$ & $8-9$ & methylene & -14 & 6 & $-13.9^{a}$ & $6^{b}, 7.7^{c}$ \\
$S_{5}$ & $10-13$ & saturated tail group R & -14 & 6 & $-13.9^{a}$ & $6^{b}, 7.7^{c}$ \\
$S_{5}$ & $10-13$ & conjugated tail group R & 3.5 & 4,1 & $5.9^{e}$ & $4^{e}, 3.6^{a}$ \\
$S_{6}$ & $14-\mathrm{N}$ & $\mathrm{Ga}_{2} \mathrm{O}_{3}$ & -4.5 to -11.5 & - & - & - \\
\hline
\end{tabular}

structures without considering the oxide layer. However, it should be noted that adding the oxide layer to the model does not change the orbital structures. We describe how we fixed the parameters for the different segments of the molecules in the supplementary information.

It may seem impossible to get the HOMO to be localized on the sulphur in view of its $\epsilon$ value being lower than that of the amide unit, a difference which would only become more pronounced taking into account the weaker coupling of the amide unit to its neighbours compared with the strong S-C coupling. The reason is that there are two orbitals localized on the sulphur, a bonding state, which corresponds to the HOMO-5, and the state which which is anti-bonding with the first $\mathrm{C}$ atom, and which forms the HOMO. Also on the amide group, there are two states, one bonding and the other anti-bonding. The weak internal coupling in this group causes the splitting between these two to be quite small, therefore they both lie below the HOMO.

\section{Results for the current}

Once the molecule is coupled to the electrodes, the retarded Green's function of the system can be obtained as $G^{R}=[\omega S-H-\Sigma]^{-1}$, where $\Sigma$ is the total self-energy obtained from 
left (L) and right (R) self-energies, $\Sigma=\Sigma^{L}+\Sigma^{R}$. Within the wide band limit (WBL), $\Sigma^{L}$ and $\Sigma^{R}$ are purely imaginary and do not depend on energy. They represent the broadenings $\Sigma^{L / R}=-\frac{i}{2} \Gamma^{L / R}$. Once the Green's functions are known, the current can be calculated from a Landauer-type equation:

$$
I=\frac{i e}{h} \int d \omega T(\omega)\left[f\left(\omega, \mu_{L}\right)-f\left(\omega, \mu_{R}\right)\right]
$$

where

$$
T(\omega)=\operatorname{Tr}\left\{\Gamma_{L} G^{r} \Gamma_{R} G^{a}\right\}
$$

is the transmission, $\mu_{L}=E_{f}-V / 2, \mu_{R}=E_{f}+V / 2$ and $G^{a}$ is the advanced Green's function which is found as the complex conjugate of $G^{r}$. The Fermi function $f(\omega, \mu)$ describes the electronic occupation of the levels, $f(\omega, \mu)=1 /\left(1+\exp \left(\frac{\omega-\mu}{k T}\right)\right)$. The $\Gamma$ parameters describe the molecule-electrode coupling and are assumed the same for all molecules in our calculation.

The only parameters that change across molecules 1-13 are the site energy and the tunneling parameters of $S_{5}$, i.e. $\varepsilon_{R}, t_{R}$ and $t_{R}^{\prime}$, representing the tail group. To study the transport through molecules 1-13, we calculate the current for different values of these three parameters. It should be noted that in matching the orbital structure from our TBTM to the DFT results, we have found it sometimes necessary to shift the site energies of the tail group somewhat (see Supplementary information). The variation of $\varepsilon_{\mathrm{R}}$ evaluated from DFT is $\sim 1 \mathrm{eV}$. Our results for molecules with conjugated and saturated tails and in the presence of a bias voltage are shown in Fig. 8. They show that the largest difference in the current is a factor of $\sim 7.6$ at bias voltage $V_{b}=0.5 \mathrm{eV}$. This result agrees well with the results of the experiment (measured at bias $0.5 \mathrm{eV}$ ). In addition, the allowed amount of variation for these parameters to reproduce the main features of the orbital structure and current is \pm 0.3 $\mathrm{eV}$.

The structures of some tail groups in Fig. 1 are not linear. Therefore, we also investigate the transport through a system with a cyclic tail group as shown in Fig. 9, which provides 


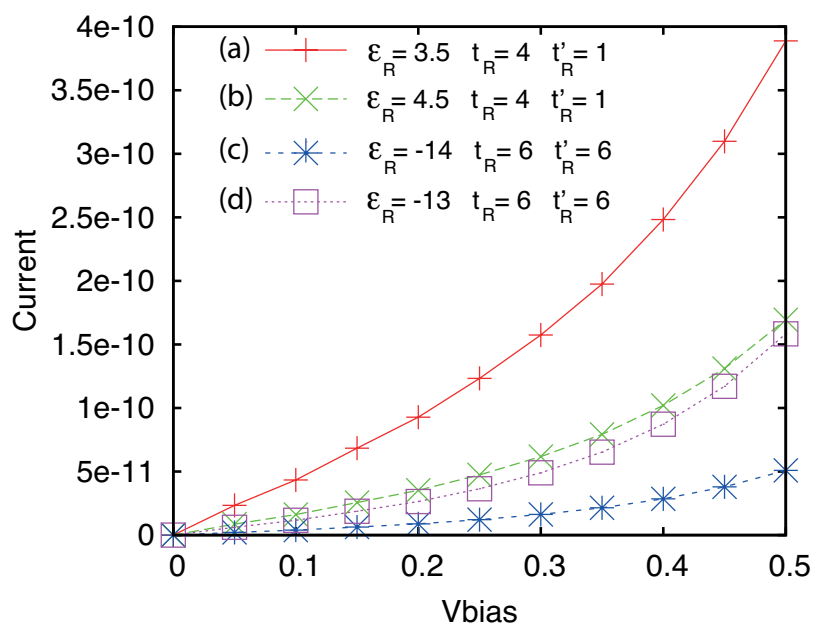

Figure 8: The current for (a), (b), (c) and (d) parameters considered Fig. 11, is shown here. The ratio of the current variation with respect to current in case (a) at voltage $V_{b}=0.5 \mathrm{eV}$ changes at most by a factor of $\sim 7.6$.

two pathways for the particles moving through the tight-binding chain. The current through such a chain is compared to the results of original model of Fig. 7 both for molecules with conjugated $\left(\varepsilon_{\mathrm{R}}=3.5, t_{\mathrm{R}}=4, t_{\mathrm{R}}^{\prime}=1\right)$ and saturated $\left(\varepsilon_{\mathrm{R}}=-14, t_{\mathrm{R}}=6, t_{\mathrm{R}}^{\prime}=6\right)$ tail groups. We emphasize that, although the difference between the site energies for pi- and sigma orbitals seems rather dramatic $(3.5$ versus $-14 \mathrm{eV})$, this does not imply a similar difference in chemical potential: as the pi-system is only half filled in the neutral state, the highest occupied pi-level is still below the $\epsilon$ for pi-sites, and for the R-groups we consider here they turn out to be about $1 \mathrm{eV}$ below the Fermi energy of the gold. The sigma orbitals are all filled and therefore reach up to $\epsilon+2 \tau \approx-14+2 \cdot 6=-2 \mathrm{eV}$. As shown in Fig. 9, the current through the molecules with cyclic structure is in the same range of molecules with linear tails, and the largest overall change between the I-V curves is a factor of 8 .

As shown in the supplementary information, the variation of $\varepsilon_{\mathrm{R}}$ evaluated from DFT is not large $(\sim 1 \mathrm{eV})$. Within this variation the highest occupied orbitals, which are largely responsible for the transport, are always localized on specific regions of the molecules. For instance, the HOMOs in all molecules are located on the thiol anchoring group. However, the shape of the tunneling barrier is determined by the entire tunneling chain which contains 

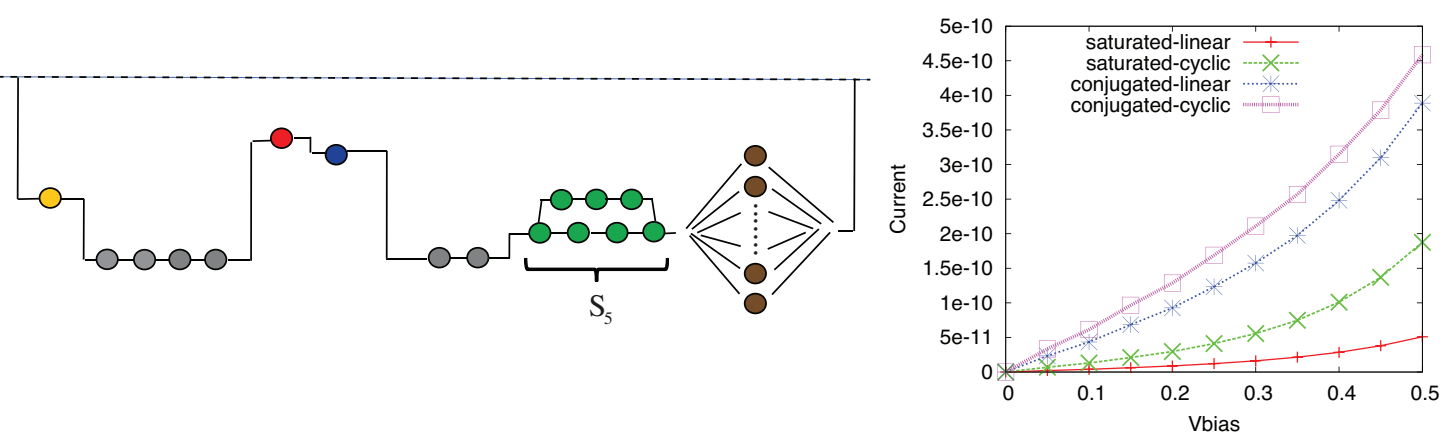

Figure 9: Current for the molecules shown in Fig. 8, but with the linear segment $S_{5}$ representing the tail group $\mathrm{R}$, replaced by a cyclic chain which provides two pathways for the electrons going through the tight-binding chain. For conjugated groups, $\varepsilon_{\mathrm{R}}=3.5, t_{\mathrm{R}}=4$, $t_{\mathrm{R}}^{\prime}=1$. For saturated groups, $\varepsilon_{\mathrm{R}}=-14, t_{\mathrm{R}}=6, t_{\mathrm{R}}^{\prime}=6$.

the tail group at the end of the chain. Therefore, the transmission through the tail group is important. Our analysis indicates that the combination of $t_{\mathrm{R}}$ and $t_{\mathrm{R}}^{\prime}$ compensates for the variation of the site energy, $\varepsilon_{\mathrm{R}}$, which is close to the Fermi energy in conjugated groups, whereas in saturated groups it is far from the Fermi energy ${ }^{2}$. In other words, the gateway from a saturated orbital to a conjugated electron system on the tail group can be viewed as a narrow passage leading to an easily traversable track. Moving from the conjugated chain to a saturated tail group is easy (the t-matrix element is large), but the orbital energy is much farther away from the Fermi energy, suppressing the current through this structure.

\section{Conclusions}

We have investigated the transport through a series of self-assembled monolayers with varied tail groups, as measured by Yoon et al. ${ }^{13}$ DFT-NEGF calculations confirm the modest current variation observed experimentally. DFT calculations show that the HOMO is largely located on the thiol group in these molecules, and this is the closest orbital to the Fermi energy of the electrodes. Therefore the transport is hole-like. To understand the weak effect of the tail group on the conductance, we have constructed a tight-binding toy model with site energies and tunneling parameters based on a series of DFT arguments and existing

\footnotetext{
${ }^{2}$ Remember that the TBTM model was not designed to yield excitation energies- see section III.
} 
literature. Our model reproduces the structure of the highest occupied orbitals (HOMO, HOMO-1, HOMO-2 and HOMO-3). Our analysis suggests a few reasons for the surprisingly small differences among the currents in these molecules with saturated and conjugated tails: (1) The location and the energy of the frontier orbitals which are responsible for the transport are similar across the entire series. For instance, the HOMO in all molecules is localized on the thiol linker. (2) The transmission is therefore mainly determined by the tunneling through the rest of the molecule, which is influenced by the tail group. In the tail groups, the differences between the values of the site energies $(\varepsilon)$ and tunneling parameters $(t)$ in the conjugated and saturated groups largely compensate each other: Conjugated groups have smaller coupling (than saturated ones) but their $\varepsilon$ is much closer to the Fermi energy than is that for saturated groups. The combination of both still leads to better conductivity of the conjugated groups. However, the coupling of the tail group to the alkane chain $\left(t_{\mathrm{R}}^{\prime}\right)$ is much weaker for conjugated groups than for saturated ones. So the combination of $t_{\mathrm{R}}$ and $t_{\mathrm{R}}^{\prime}$ compensates for the large difference between $\varepsilon$ 's in conjugated and saturated groups. (3) The conductance is also influenced by the broadening of the molecular levels connected to the left and right electrodes $\left(\Gamma_{L, R}\right)$. In all these molecules $\Gamma_{L}$ is the same and $\Gamma_{R}$ is determined by the connection to the oxide layer. For the two molecules $\mathrm{C}_{12}$ and $\mathrm{C}_{18}$ (used as calibration standards) in which the length of the alkane tail group is extended, the coupling to the electrode decays exponentially, and therefore a significant change in the I-V characteristic can be observed. We found that the decay constant is $\beta=1.1$ per methylene group in alkanethiols which in agreement with the experimental results $(\beta=0.9-1.0)$. We therefore expect the similarity of the current for saturated and conjugated tail groups to depend sensitively on the tail group length - longer tail groups would lead to saturated tail groups yielding lower current densities. 


\section{Acknowledgement}

F. M. gratefully acknowledges the hospitality of the M. A. R. group in Northwestern University and useful discussions with J. S. Seldenthuis and B. Movaghar. We thank H. J. Yoon for sharing the data of the measured current with us. Financial support was obtained from the EU FP7 program under the grant agreements SINGLE and ELFOS. Collaborative aspects of the research involving M. A. R. and G. M. W. were supported by the non-equilibrium energy research center (NERC), an Energy Frontier Research Center funded by the U.S. Department of Energy Office of Science, Office of Basic Energy Sciences under award II DE-SC0000989. M. A. R thanks the Chem. Division of the NSF (CHE1058896) for support.

\section{Contributions}

F. M. did the computations and drafted the manuscript. J. M. T. and M. A. R. created the tight-binding model and edited the manuscript. G. M. W. suggested the project, provided the experimental data, and edited the manuscript. 


\section{Supporting Information}

Here we present a few details concerning the determination of the tight-binding parameters. In particular we discuss how we obtain these parameters and we show how these parameters can reproduce the structure of molecular orbitals:

\section{(A) Finding the tight-binding parameters}

We find most of the parameters of the Hamiltonian from the results shown for molecular orbitals and from calculations on periodic chains as follows:

- Alkane segment and saturated tail group: The parameters for an alkane chain should satisfy three criteria: (I) From several NEGF calculations, ${ }^{4,16}$ it is established that the HOMO is located about $2 \mathrm{eV}$ below the Fermi energy of the metal. (II) The conductance through alkane chains decreases exponentially with molecular length with the exponential rate of $\beta \sim 1$ per methylene group, ${ }^{15-19,23}$ i.e. $G(L)=G_{0} e^{-\beta L}$. (III) The energy separation between the levels, in particular the frontier orbitals, should be in agreement with that found in DFT. This separation is determined (for a homogeneous chain) by the parameter $t$, as the levels are distributed according to $E_{n}-\varepsilon=-2 t \cos \left(k_{n} a\right)$ where $k_{n}=n \pi / L$. Here $a$ is the inter-site distance and $L$ is the effective chain length. From the orbitals found in DFT, we use those that have significant sigma character. The higher orbitals are easily identified as such - the lower ones tend to hybridize with s-orbitals on the hydrogen atoms. These three criteria together lead to a tunneling parameter $t_{\mathrm{A}}=6 \mathrm{eV}$ and a site energy $\varepsilon_{\mathrm{A}}$ of $-14 \mathrm{eV}$. This value for $\varepsilon$ seems very low. However together with the coupling with a value of $t$ about 6 , this leads to a level at $\varepsilon+2 t \sim-2 \mathrm{eV}$ for a long alkane chain.

Our parameters yield $\beta=1.1$ per $\mathrm{CH}_{2}$ unit based on the analytical formula $\beta=$ $\cosh ^{-1}\left(-\frac{E_{F}-\varepsilon-2 t}{2 t}\right)$ which follows directly from the independent-electron Schrödinger equation.

- Amide group: We have performed a DFT calculation for an alternating $\left[\mathrm{CH}_{2}-\text { amide }\right]_{n}$ 
chain (with length $n=8$ ). The results reveal that the bandwidth of the amide states is about $2 \mathrm{eV}$. We get a similar bandwidth in our model for the parameters $t_{\text {amide }}=1.8 \mathrm{eV}$, $t_{\text {amide }}^{\prime}=0.5 \mathrm{eV}, \varepsilon_{\mathrm{CO}}=-1.3 \mathrm{eV}$ and $\varepsilon_{\mathrm{N}}=-1.8 \mathrm{eV}$, which can also reproduce the structure of the highest occupied orbitals, HOMO, HOMO-1, HOMO-2 and HOMO-3.

- Conjugated R tail group: (I) Based on Xu et al. ${ }^{40}$ for $1.4 \AA$ pi carbon bonds, and the HOMO-LUMO gap of DFT calculations for conjugated groups, we choose the tunneling parameter, $t_{R}=4 \mathrm{eV}$ for this tail group. (II) $\varepsilon_{\mathrm{R}}$ for a conjugated tail is chosen to be 3.5 $\mathrm{eV}$ to reproduce the general structure and arrangement of the orbitals. The deviation from the literature value is due to charge transfer at molecule-metal interface shifting the HOMO down. Moreover, $t_{\mathrm{R}}^{\prime}=1 \mathrm{eV}$ as obtained from DFT calculations of a molecule consisting of an alkane chain connected to a conjugated part such as benzene, and is also in agreement with the one obtained by Benkö et al. ${ }^{39}$

- Thiol group: We take for the site energy and tunneling amplitude the values $\varepsilon_{\mathrm{S}}=-4.2$ $\mathrm{eV}$ and $t_{\mathrm{A}}=6 \mathrm{eV}$ to localize the HOMO on its own site and with a small weight on the amide group.

- Oxide layer: Both experiments ${ }^{44}$ and DFT calculations ${ }^{44}$ show that for the oxide layer the bandwidth of the valence band is about $7 \mathrm{eV}$. The parameters chosen for the top and the bottom of the band of the oxide layer are therefore $\varepsilon_{\mathrm{Ox}_{\mathrm{t}}}=-4.5 \mathrm{eV}$ and $\varepsilon_{\mathrm{Ox}_{\mathrm{b}}}=-11.5$ $\mathrm{eV}$ respectively. All sites representative of the oxide layer are coupled to the last site of the tail group by $t_{\mathrm{Ox}}=0.1 \mathrm{eV}$.

The results presented below are valid for a range of values of these parameters- see supporting information for details.

In matching the orbital structure from our TBTM to the DFT results shown in Fig. 6 and Fig. 12, we have found it sometimes necessary to shift the site energies of the tail group somewhat. To obtain a rough estimate of the variation of the potential $\varepsilon_{\mathrm{R}}$ of the tail group, we performed DFT calculations for the isolated tail groups (i.e, tail groups in gas phase with a single hydrogen instead of the attached alkane chain); see Fig. 10. We then take 
the resulting variation of the HOMO level as an indication of the variation of the tail group site energy. Our DFT calculations show that the variation of the HOMO energies is $\sim 1$ $\mathrm{eV}$ both in conjugated and in saturated groups. The fact that this potential varies so little from molecule to molecule is largely responsible for the small variation in the current. It should be noted that the HOMO energy in molecules 8-12 is closer to the Fermi energy of the electrodes than in molecule 13 which is due to their larger number of atoms and consequently broader band.
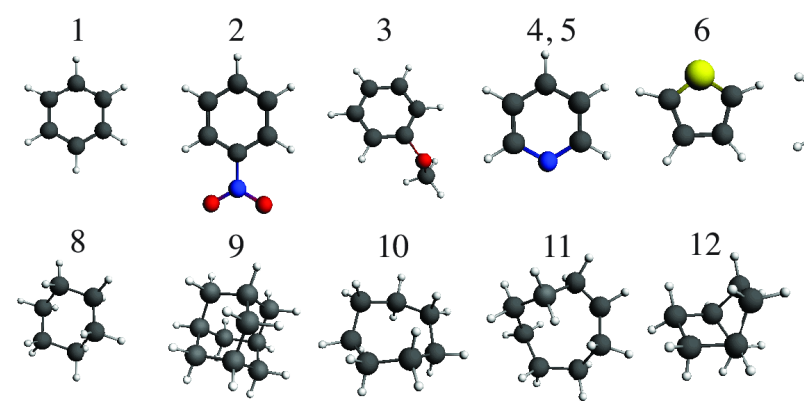

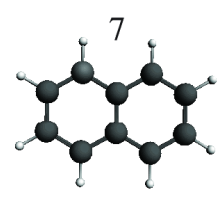

13

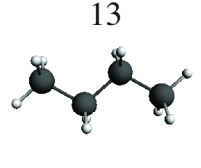

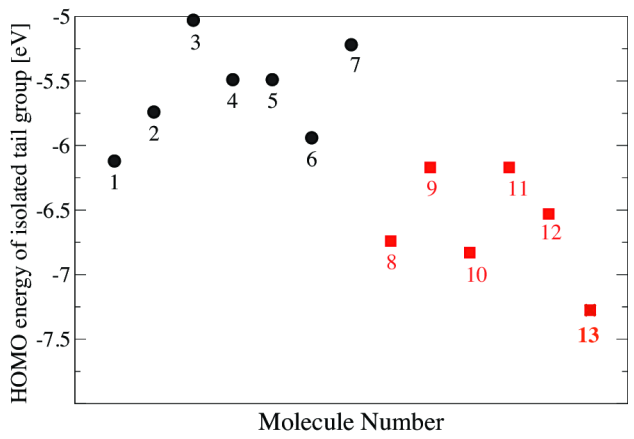

Figure 10: The HOMO energy for the isolated tail groups (with a single hydrogen instead of the attached alkane chain). The HOMO energy for the conjugated tail groups (molecules $1-7)$, is in the range $[-5.05,-6.1] \mathrm{eV}$. The HOMO energy for the aliphatic tail groups in molecules $8-12$ is in the range $[-6.17,-6.8] \mathrm{eV}$.

\section{(B) Molecular orbitals}

Using the parameters described in the previous section, we now present the structures of orbitals obtained for our TBTM. Changing the site energy of the tail group $\left(\varepsilon_{\mathrm{R}}\right)$ and its tunneling coupling $t_{\mathrm{R}}$ and $t_{\mathrm{R}}^{\prime}$ (see Fig. 7 ) for molecules with conjugated and saturated tails does not influence the HOMO energy located on the thiol linker. To verify this conclusion, we consider the electron population of a few of the highest eigenstates in the TBTM of Fig. 7.

For the molecular orbital $\psi_{n}=\sum_{i} C_{i, n} \phi_{i}$, where the sum is over the 17 different sites, the population of site $i$ is $\left|C_{i, n}\right|^{2}$. These populations for the HOMO, HOMO-1, HOMO-2 and HOMO-3 are shown in Fig. 11 for molecules with conjugated $\left(\varepsilon_{\mathrm{R}}=3.5 \mathrm{eV}\right.$ and $\left.t_{\mathrm{R}}=4 \mathrm{eV}\right)$ 
and saturated $\left(\varepsilon_{\mathrm{R}}=-14.0 \mathrm{eV}\right.$ and $\left.t_{\mathrm{R}}=6 \mathrm{eV}\right)$ tails. The structure of the HOMO orbitals is in agreement with our DFT calculations shown in Fig. 6 and Fig. 12. Furthermore, the lower levels such as HOMO-1, HOMO-2, HOMO-3 of the TBTM are mostly localized on amide (plus tail group in some conjugated molecules), the tail group $\mathrm{R}$ or on both. Due to a proper choice of parameters, their structure is in agreement with our DFT calculations.

We now show how the orbitals vary when we alter $\varepsilon_{\mathrm{R}}$. We take this variation within 1 $\mathrm{eV}$ (as implied by Fig. 10), both for saturated and conjugated tail groups. For molecules with a saturated tail group, shifting the site energy to $\varepsilon_{\mathrm{R}}=-13 \mathrm{eV}$ does not change the structure of the orbitals, but for molecules with a conjugated tail, shifting the site energy from $\varepsilon_{\mathrm{R}}=3.5$ to $\varepsilon_{\mathrm{R}}=4.5 \mathrm{eV}$ changes the HOMO-2 and HOMO-3 slightly. For $\varepsilon_{\mathrm{R}}=4.5$ eV, HOMO-2 and HOMO-3 are both distributed over the amide and tail group whereas for $\varepsilon_{\mathrm{R}}=3.5 \mathrm{eV}$, HOMO-2 is on the amide group and HOMO-3 is localized on the $\mathrm{R}$ group. These agree well with our DFT results.

These results show that with these selected parameters we can roughly reproduce the structure of the frontier orbitals. The allowed amount of variation for these parameters to reproduce the main features of the orbital structure and current, is about $\pm 0.3 \mathrm{eV}$. 

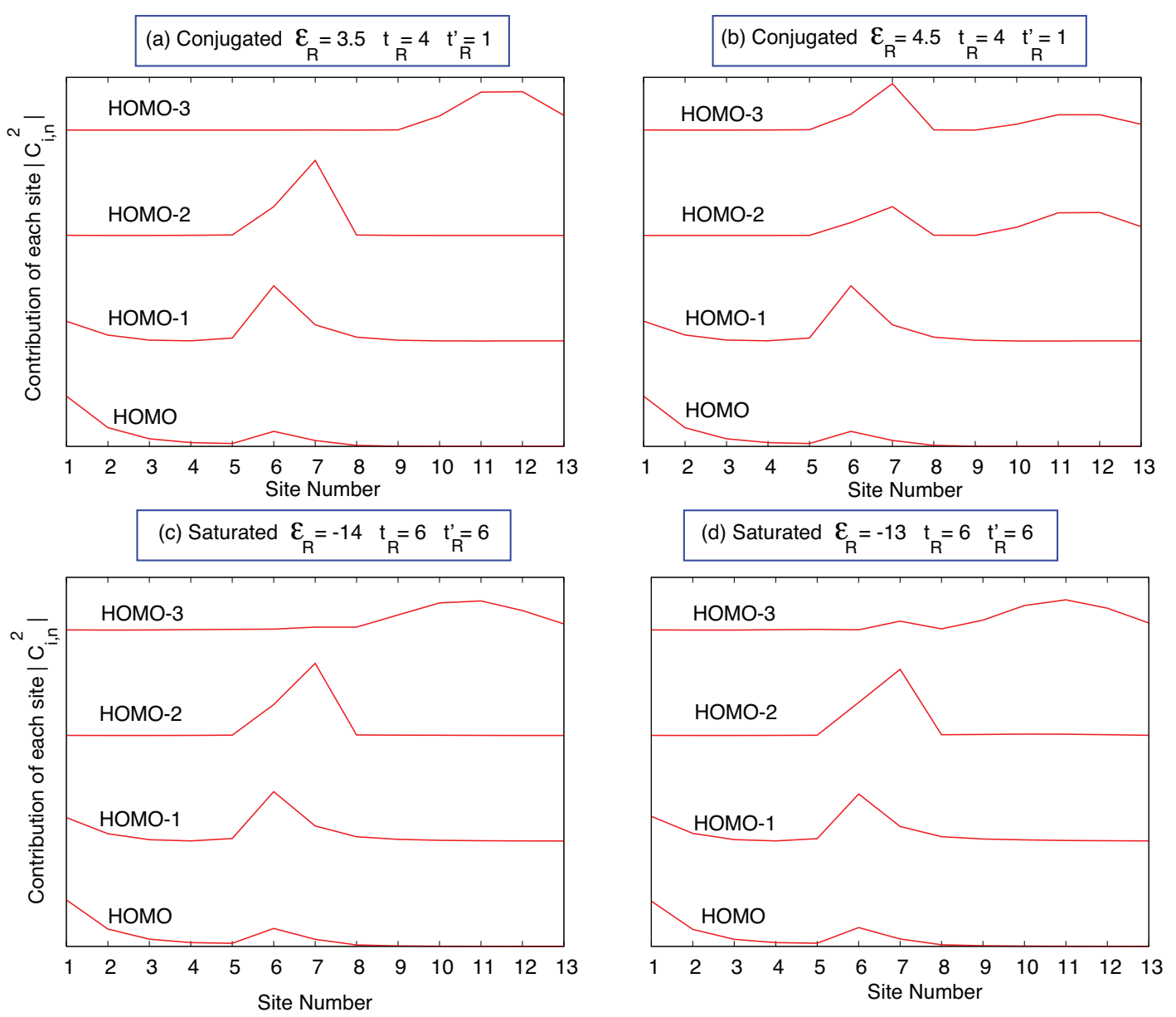

Figure 11: Contribution $\left|C_{i, n}\right|^{2}$ of each site $i$ to the electron population for each orbital n, $n=$ HOMO, HOMO-1, HOMO-2 and HOMO-3 levels. Site 1 is the thiol group and sites 6-7 are the amide group. Sites 10-13 are the tail groups. (a) Molecules with conjugated tail groups $\varepsilon_{\mathrm{R}}=3.5 \mathrm{eV}, t_{\mathrm{R}}=4 \mathrm{eV}$ and $t_{\mathrm{R}}^{\prime}=1 \mathrm{eV}$. (b) Molecules with conjugated tail, $\varepsilon_{\mathrm{R}}$ shifted by $1 \mathrm{eV}$ to see how the variation influences the orbitals. (c) Molecules with saturated tail groups $\varepsilon_{\mathrm{R}}=-14.0 \mathrm{eV}, t_{\mathrm{R}}=6 \mathrm{eV}$ and $t_{\mathrm{R}}^{\prime}=6 \mathrm{eV}$. (d) Molecules with saturated tail, $\varepsilon_{\mathrm{R}}$ shifted by $1 \mathrm{eV}$ to see how the variation influences the orbitals. Note that the difference between (a) and (b) for HOMO-2 and HOMO-3 nicely corresponds to Fig. 12. 


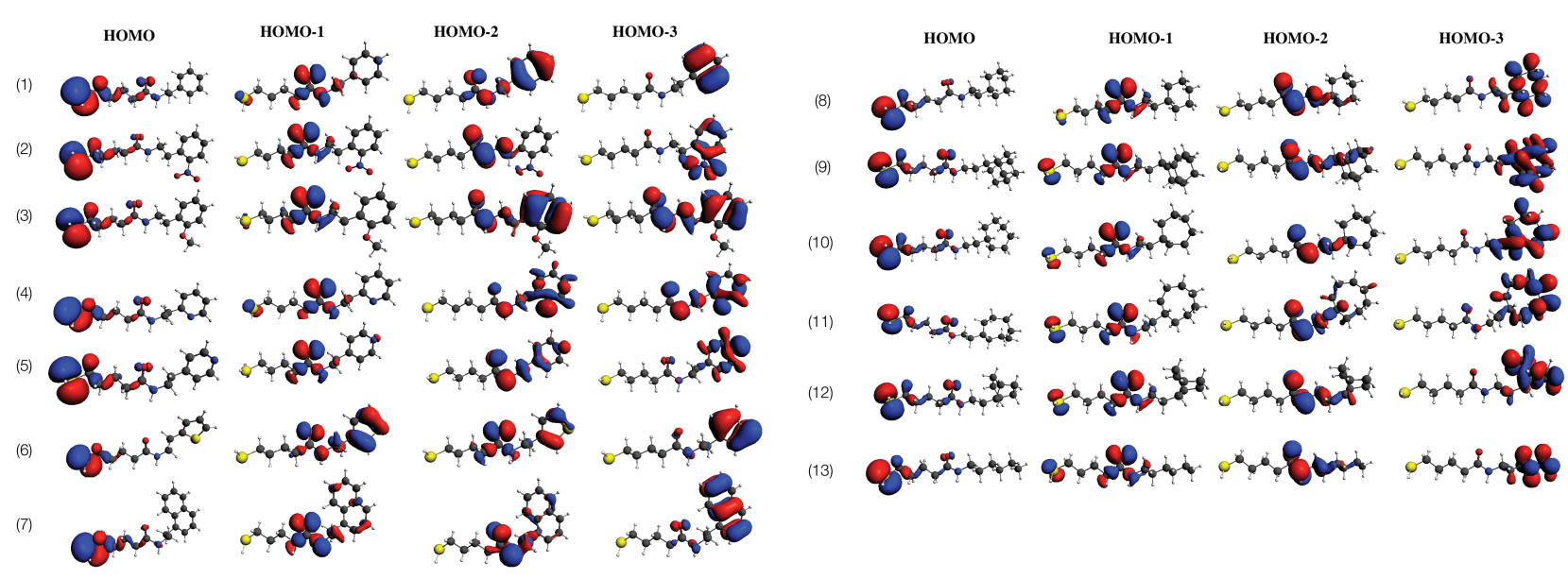

Figure 12: HOMO, HOMO-1, HOMO-2 and HOMO-3 of the molecules 1-13 are mostly located on thiol, amide, amide (plus tail group $\mathrm{R}$ ), and tail group $\mathrm{R}$ respectively. These orbitals are to be compared with their counterparts from the TBTM in Fig. 11.; the TMTB indeed mimics the full DFT results very closely.

\section{References}

(1) Nitzan, A.; Ratner, M. A. Science 2003, 300, 1384.

(2) Paulsson, M.; Zahid, F.; Datta, S. Resistance of a molecule; CRC Press, 2003; chapter in Handbook of Nanotechnology, edited by W. A. Goddard III, D. W. Brenner, S. E. Lyshevski, and G. J. Iafrate.

(3) Datta, S. Quantum Transport: Atom to Transistor; Cambridge University Press, 2005.

(4) Cuevas, J. C.; Scheer, E. Molecular electronics, an introduction to theory and experiment; World Scientific, 2010.

(5) Mujica, V.; Ratner, M. A. Chem. Phys. 2001, 264, $365-370$.

(6) Galperin, M.; Ratner, M. A.; Nitzan, A.; Troisi, A. Science 2008, 319, 1056-1060.

(7) Rampi, M. A.; Whitesides, G. M. Chem. Phys. 2002, 281, 373 - 391.

(8) Heersche, H. B.; Lientschnig, G.; O’Neill, K.; van der Zant, H. S. J.; Zandbergen, H. W. Appl. Phys. Lett. 2007, 91, 072107. 
(9) Park, H.; Lim, A. K. L.; Alivisatos, A. P.; Park, J.; McEuen, P. L. Appl. Phys. Lett. 1999, 75, 301-303.

(10) Fracasso, D.; Valkenier, H.; Hummelen, J. C.; Solomon, G. C.; Chiechi, R. C. JACS 2011, 133, 9556-9563.

(11) Arroyo, C.; Frisenda, R.; Moth-Poulsen, K.; Seldenthuis, J.; Bjornholm, T.; van der Zant, H. Nanoscale Research Letters 2013, 8, 234.

(12) George, C.; Yoshida, H.; Goddard, W. A.; Jang, S. S.; Kim, Y.-H. J. Phys. Chem. B 2008, 112, 14888-14897.

(13) Yoon, H. J.; Shapiro, N. D.; Park, K. M.; Thuo, M. M.; Soh, S.; Whitesides, G. M. Angew. Chem. Int. Ed. 2012, 51, 4658-4661.

(14) Dickey, M. D.; Chiechi, R. C.; Larsen, R. J.; Weiss, E. A.; Weitz, D. A.; Whitesides, G. M. Advanced Functional Materials 2008, 18, 1097-1104.

(15) Lee, T.; Wang, W.; Klemic, J. F.; Zhang, J. J.; Su, J.; Reed, M. A. J. Phys. Chem. B. 2004, 108, 8742-8750.

(16) Li, C.; Pobelov, I.; Wandlowski, T.; Bagrets, A.; Arnold, A.; Evers, F. JACS 2008, $130,318-326$.

(17) Xu, B.; Tao, N. J. Science 2003, 301, 1221-1223.

(18) Slowinski, K.; Chamberlain, R. V.; Miller, C. J.; Majda, M. JACS 1997, 119, 1191011919.

(19) Smalley, J. F.; Feldberg, S. W.; Chidsey, C. E. D.; Linford, M. R.; Newton, M. D.; Liu, Y.-P. J. Phys. Chem. 1995, 99, 13141-13149.

(20) Wold, D. J.; Frisbie, C. D. JACS 2000, 122, 2970-2971. 
(21) Bumm, L. A.; Arnold, J. J.; Dunbar, T. D.; Allara, D. L.; Weiss, P. S. J. Phys. Chem. B. 1999, 103, 8122-8127.

(22) Kaun, C.-C.; Guo, H. Nano Lett. 2003, 3, 1521-1525.

(23) Akkerman, H. B.; de Boer, B. J. Phys: Condens. Matter 2008, 20, 013001.

(24) Fagas, G.; Delaney, P.; Greer, J. C. Phys. Rev. B 2006, 73, 241314.

(25) Xue, Y.; Datta, S.; Ratner, M. A. Chem. Phys. 2002, 281, $151-170$.

(26) Brandbyge, M.; Mozos, J.-L.; Ordejón, P.; Taylor, J.; Stokbro, K. Phys. Rev. B 2002, $65,165401$.

(27) Verzijl, C. J. O.; Seldenthuis, J. S.; Thijssen, J. M. J. Chem. Phys. 2013, 138, 094102.

(28) Jauho, A.-P.; Wingreen, N. S.; Meir, Y. Phys. Rev. B 1994, 50, 5528-5544.

(29) Lawson, J. W.; Bauschlicher, C. W. Phys. Rev. B 2006, 74, 125401.

(30) ADF2010, SCM, Theoretical Chemistry, Vrije Universiteit, Amsterdam, The Netherlands, http://www.scm.com.

(31) Shao, Y. et al. Phys. Chem. Chem. Phys. 2006, 8, 3172-3191.

(32) Hoffmann, R. The Journal of Chemical Physics 1963, 39, 1397-1412.

(33) Mulliken, R. S.; Rieke, C. A.; Orloff, D.; Orloff, H. J. Chem. Phys. 1949, 17, 1248-1267.

(34) Jasper, A. W.; Schultz, N. E.; Truhlar, D. G. J. Chem. Theory Comput. 2007, 3, $210-218$.

(35) Hoffmann, R. J. Chem. Phys. 1963, 39, 1397-1412.

(36) Pariser, R.; Parr, R. G. J. Chem. Phys. 1953, 21, 466-471.

(37) Pople, J.; Beveridge, D. Approximate Molecular Orbital Theory; McGraw-Hill, 1970. 
(38) Dewar, M. J. S.; Thiel, W. JACS 1977, 99, 4899-4907.

(39) Benkö, G.; Flamm, C.; Stadler, P. F. J. Chem. Inf. Comput. Sci. 2003, 43, 1085-1093, PMID: 12870897.

(40) Xu, C. H.; Wang, C. Z.; Chan, C. T.; Ho, K. M. J. Physics: Condens. Matter 1992, 4, 6047 .

(41) Wang, Y.; Mak, C. Chem. Phys. Lett. 1995, 235, $37-46$.

(42) Murrell, J. N. The theory of the electronic spectra of organic molecules; John Wiley and Sons Inc, New York, Methuen and CO LTD, London, 1963.

(43) Salem, L. Molecular orbital theory of conjugated systems; W.A. Benjamin, Inc., New York, 1966.

(44) Janowitz, C.; Scherer, V.; Mohamed, M.; Krapf, A.; Dwelk, H.; Manzke, R.; Galazka, Z.; Uecker, R.; Irmscher, K.; Fornari, R.; Michling, M.; Schmeißer, D.; Weber, J. R.; Varley, J. B.; de Walle, C. G. V. New J. Phys. 2011, 13, 085014. 